\title{
La banca digital: Innovación tecnológica en la inclusión financiera en el Perú
}

Allan Herminio Vargas Garcia ${ }^{1}$

\begin{abstract}
RESUMEN
El impacto de la transformación digital impulsado por los avances tecnológicos afecta al sector financiero con la entrada de nuevos modelos de negocios como las fintech y bigtech que compiten con éxito ofreciendo productos financieros con potencial disruptivo. La banca digital es el resultado de la suma de la banca tradicional y el internet donde se ofrecen servicios bancarios a través de una página web o aplicación móvil. La integración de servicios financieros en ecosistemas digitales representa un reto para la banca tradicional que necesita evolucionar para enfrentar los cambios del entorno. La banca digital cumple un papel importante en la inclusión financiera, pues permite que más peruanos tengan acceso a servicios innovadores e impulsa el crecimiento económico. Sin embargo, también existe un alto nivel de fraude digital cuyo objetivo es hurtar dinero o chantajear a los usuarios financieros mediante el phishing y el uso de malware. Para evaluar las variables "banca digital" e "inclusión financiera" se utilizó el coeficiente de correlación de Pearson, útil para relaciones lineales, y para la tabulación de los resultados se utilizó el software SPSS-IBM.
\end{abstract}

Palabras clave: innovación; tecnología; inclusión; finanzas, banca.

\section{INTRODUCCIÓN}

La presente investigación estudia la importancia de las tecnologías empleadas por la banca digital, las cuales ofrecen una importante alternativa para integrar a la población, excluida socialmente, a través del acceso y uso de servicios financieros (inclusión financiera). Estas, empezando por el tráfico de datos en teléfonos móviles, transacciones interbancarias y cuentas digitales, pueden utilizarse como herramientas para aumentar la eficiencia en el servicio de atención al cliente.

La inclusión financiera es un concepto de carácter multidimensional del lado de la oferta y la demanda de productos o servicios financieros, cuyas dimensiones son el acceso, el uso, la calidad y el impacto sobre el bienestar financiero de las familias y las empresas. Según la Comisión Económica para América Latina y el Caribe (CEPAL), la inclusión financiera es un factor que impulsa 7 de los 17 objetivos de desarrollo sostenible: "(ODS1) Fin de la pobreza, (ODS2) Hambre cero, (ODS3) Salud y bienestar, (ODS5) Igualdad de género, (ODS8) Trabajo decente y crecimiento económico, (ODS9) Industria, innovación e infraestructura y (ODS10) Reducción de las desigualdades" (CEPAL, 2020, p.14), por lo que esta investigación adquiere especial relevancia. El objetivo de este estudio es determinar en qué medida la banca digital se relaciona con la inclusión financiera durante el periodo 2010-2019, mientras que la hipótesis general busca validar que la banca digital se relaciona significativamente con la inclusión financiera en el Perú durante el periodo 2010-2019.

Esta investigación contribuye a expandir el conocimiento en torno al cambio que la innovación tecnológica está generando en el uso de los servicios financieros y el acceso a ellos, lo que implica que el futuro de la banca y el desarrollo de la inclusión financiera es digital.

1 Magíster en Administración por la Universidad del Pacífico. Actualmente, es docente permanente de la Facultad de Ciencias Administrativas de la Universidad Nacional Mayor de San Marcos. (Lima, Perú).

E-mail: avargasg@unmsm.edu.pe 


\section{La digitalización en América Latina}

En términos de desarrollo digital, América Latina se ubica en un nivel medio con un índice de 49.92 (en una escala de 0 a 100), superior a la región de África (35.05) y Asia Pacífico (49.16). Sin embargo, muestra un rezago respecto a Europa del Este (52.90), Europa Occidental (71.06) y América del Norte (80.85). América Latina, como parte del mundo emergente, presenta una moderada tasa de crecimiento de digitalización respecto a otras regiones del mundo desarrollado (Corporacion Andina de Fomento [CAF], 2020a).

El índice de preparación para las tecnologías de vanguardia realizado por la Organización de las Naciones Unidas [ONU] (2020) mostró los resultados obtenidos por 158 países basándose en una escala de puntuaciones de 0 a 1 ubicándolos dentro de uno de los cuatro grupos de puntuación del percentil 25. Los valores del índice considerados fueron: bajo, medio-bajo, medio-alto y alto. En la Tabla 1 se muestra la posición obtenida por los países representativos de América Latina.

Tabla 1. Índice de tecnologías en América Latina.

\begin{tabular}{lccc}
\hline \multicolumn{1}{c}{ País } & Ranking mundial & Score & Grupo \\
\hline Brasil & 41 & 0.65 & Medio-alto \\
Chile & 49 & 0.57 & Medio-alto \\
México & 57 & 0.54 & Medio-alto \\
Argentina & 65 & 0.49 & Medio-alto \\
Uruguay & 68 & 0.47 & Medio-alto \\
Colombia & 78 & 0.44 & Medio-alto \\
Perú & 89 & 0.36 & Medio-bajo \\
Ecuador & 90 & 0.34 & Medio-bajo \\
Paraguay & 102 & 0.29 & Medio-bajo \\
Bolivia & 116 & 0.24 & Medio-bajo \\
\hline
\end{tabular}

Fuente: Adaptado de la Organización de las Naciones Unidas, 2020.

\section{Inclusión financiera}

La inclusión financiera propicia el crecimiento económico centrándose en la población vulnerable, ya que facilita el ahorro, la inversión, los créditos y seguros mediante el uso y acceso a los servicios financieros. Entre las diferencias económicas y sociales de los países de Europa de altos ingresos y los países de América Latina de bajos ingresos se encuentra el uso y acceso a los servicios financieros (Orazi, Martínez, y Vigier, 2019).
Asimismo,

Tener acceso a una cuenta de transacciones es un primer paso hacia una inclusión financiera más amplia, ya que permite a las personas guardar dinero y enviar y recibir pagos. Una cuenta de transacciones también puede servir como puerta de acceso a otros servicios financieros. Por ello, garantizar que las personas de todo el mundo puedan tener acceso a una cuenta de transacciones es el centro de atención de la Iniciativa de Acceso Universal a Servicios Financieros para 2020 del Grupo Banco Mundial. (Banco Mundial, 2018, párr. 2)

En el año 2019, respecto al número de cuentas de depósito en bancos comerciales, México contó con 98 millones 623 mil, Colombia con 75 millones 728 mil, Argentina con 67 millones 579 mil, Chile con 44 millones 994 mil, Perú con 42 millones 662 mil y finalmente Bolivia con 6 millones 256 mil (Figura 1).

Conceptos como el ahorro o el acceso al crédito son aspectos importantes de la inclusión financiera. En el año 2019, respecto a cuentas de préstamos en bancos comerciales, Colombia contó con 24 millones 463 mil, Argentina con 18 millones 997 mil, Chile con 15 millones 675 mil, Perú con 5 millones 923 mil y finalmente Bolivia con 692 mil (Figura 2).

La inclusión financiera digital representa una oportunidad para que las familias de bajos ingresos que se encuentran desatendidas se integren al sector bancario y accedan a los servicios financieros digitales mediante el internet.
"Inclusión financiera digital" puede definirse como el acceso digital a servicios financie- ros formales y el uso de tales servicios por parte de poblaciones excluidas del sistema financiero y subatendidas por las institu- ciones del sector. Esos servicios deberían estar adaptados a las necesidades de los clientes y prestarse de modo responsable, a un costo que sea, al mismo tiempo, ase- quible para los clientes y sostenible para los prestadores. (Grupo Consultivo de Ayuda a los Pobres [CGAP], 2015, p. 1)

\section{Banca digital}

La aparición de tecnologías disruptivas ha llevado a la banca tradicional a ser más competitiva y a) ser rápida y precisa en la evaluación de riesgos; b) garantizar la seguridad y protección de datos de los clientes; c) explotar la inteligencia de datos 


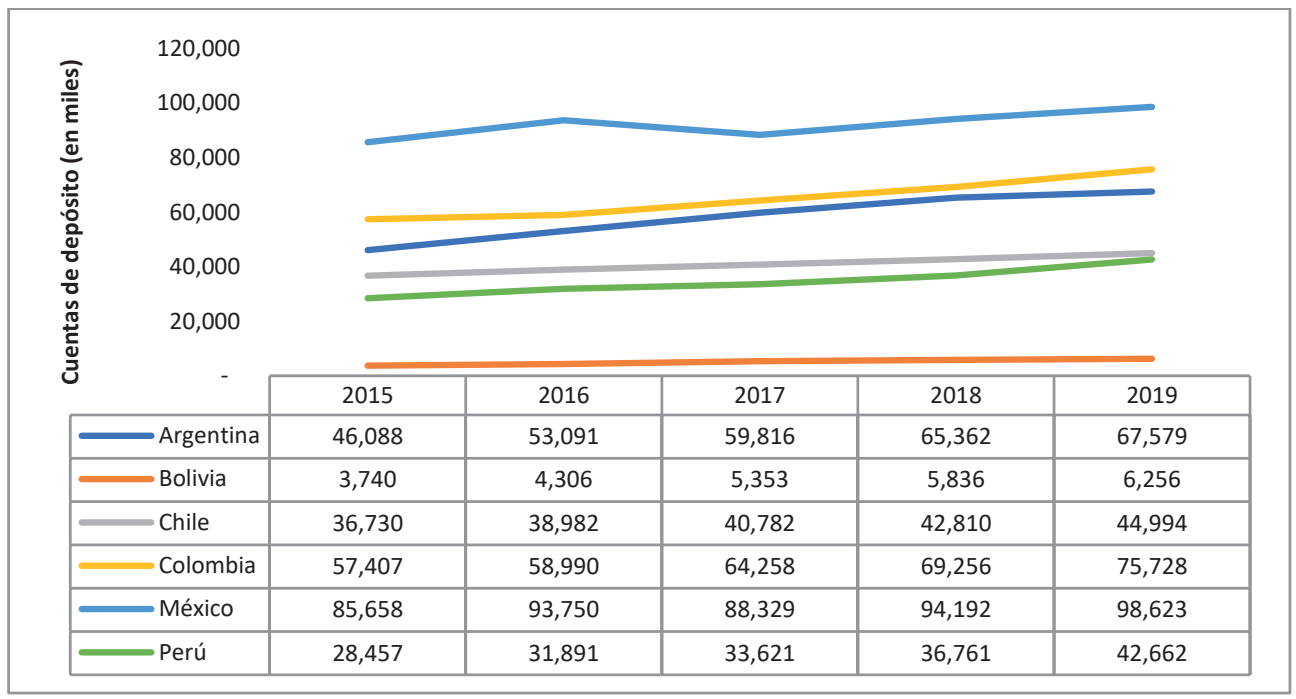

Figura 1. América Latina: cuentas de depósito en bancos comerciales 2015-2019 (en miles). Fuente: Adaptado de Fondo Monetario Internacional, 2020.

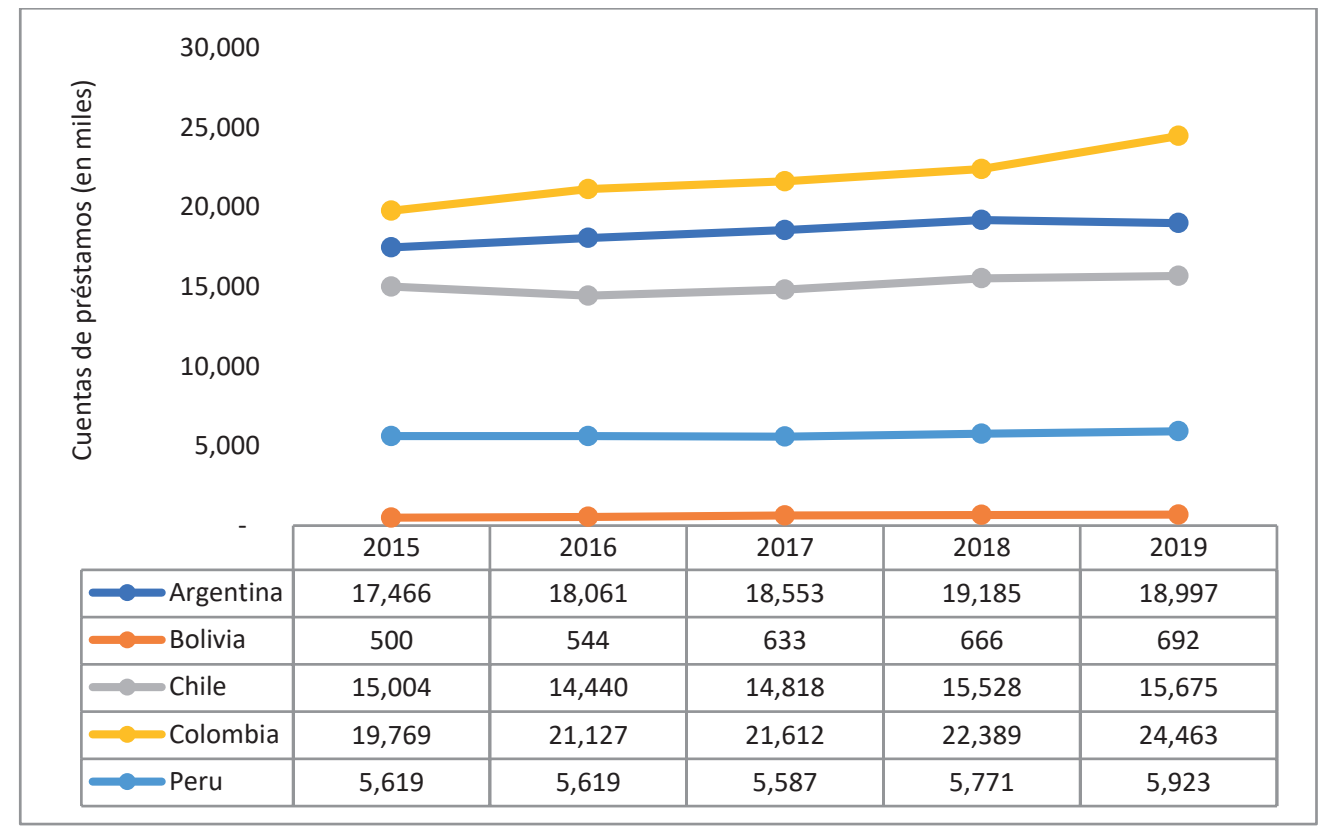

Figura 2. América Latina: cuentas de préstamos en bancos comerciales 2015-2019 (en miles). Fuente: Adaptado de Fondo Monetario Internacional, 2020.

masivos (big data) para generar valor de los usuarios; y d) ofrecer productos y servicios de acuerdo a la realidad y necesidad de los clientes digitales (Bueno, Longo, y Morcillo, 2017).

Una definición de banca digital:

(...) el mundo digital ha cambiado la dinámica tradicional de la banca, ofreciendo productos enfocados en el consumidor y sus necesidades. Se trata de un consumidor que demanda transacciones en tiempo real, seguras y eficientes, $y$ al que las nuevas tecnologías lo empoderan para: a) administrar sus productos a través de plataformas a las que pueden acceder desde cualquier lugar y en cualquier momento; b) conocer de primera mano la oferta de productos y servicios; c) comparar las opciones que le ofrece el mercado y d) emitir opiniones y defender 
sus derechos de manera más efectiva. Otra faceta de las nuevas tecnologías consiste en el impacto potencial que tienen para impulsar la inclusión financiera, particularmente en sectores de bajos ingresos. (Asobancaria, 2017, p.2)

\section{Tecnologías utilizadas por la banca digital}

\section{Big data (datos masivos)}

Es la recopilación y administración masiva de información que hace posible el almacenamiento, procesamiento y análisis de información digital. Permite a la banca tomar decisiones de manera efectiva, dado que, al conocer mejor a sus clientes, puede ofrecer productos y servicios financieros diseñados según sus necesidades. Mejora los procesos de aprobación de créditos, gestión de riesgos, banca de inversión y ventas cruzadas. Además, elimina las barreras de la asimetría de información.

El término Big Data no sólo hace referencia al volumen de datos, sino a las tecnologías asociadas a la captación, administración y visibilidad de los mismos; y no sólo se tiene en cuenta el volumen sino la variedad de los mismos y la velocidad de acceso y procesamiento. (Revuelta, 2018, p.312)

\section{Blockchain (cadena de bloques)}

Es una cadena de bloques o registro de datos descentralizado. Se trata de una base de datos compartida por una red distribuida que utiliza algoritmos encriptados haciendo de la información difícil de manipular o robar. En la banca, en un futuro cercano, facilitará la transmisión de documentos (datos) y capitales de una forma segura utilizando códigos encriptados. Asimismo, permitirá proporcionar a los clientes una identidad segura en la red; además, se reemplazarán los contratos tradicionales por los smart contract, lo que reducirá el riesgo en las transacciones.

En cuanto al sector bancario y financiero, si bien es cierto que el uso de esta tecnología aún es incipiente, algunos bancos han venido trabajando de manera importante en la utilización del blockchain en el desarrollo de sus productos, viendo en su utilización enormes posibilidades para la reducción de costos, mayor eficiencia y agilidad en las transacciones. (Federación Latinoamericana de Bancos [FELABAN], 2018, p.1)

\section{Cloud Computing (computación en la nube)}

Es la tecnología que facilita el acceso remoto por medio de internet para el almacenamiento de datos, plataformas, aplicaciones y servicios en un espacio virtual. Las nubes (servidores localizados en la red) son los entornos donde se ejecutan las cargas de trabajo. El almacenamiento de datos y servicios en la nube asegura el procesamiento rápido de transacciones y genera ahorros tanto para el cliente financiero como para la banca, genera escalabilidad y agilidad en el negocio bancario y es amigable con otras tecnologías como la inteligencia artificial y el big data.

\section{Inteligencia artificial}

Es una especialidad de la ciencia informática que se refiere al desarrollo de los sistemas para realizar tareas complejas como lo haría la inteligencia humana. La inteligencia artificial impacta en actividades de la banca como la gestión de riesgos, calificación de créditos, fraudes en tarjetas de crédito, transacciones de efectivo y el asesoramiento personalizado (app chatbot). "Un chatbot es un software que interactúa con el usuario mediante una interfaz de conversación, diseñado para simular una conversación inteligente sin un ser humano presente" (Serrano-Cobos, 2016, p.845).

La inteligencia artificial se ha convertido en una herramienta imprescindible en los mercados financieros de inversión, por lo que su uso se generalizará en el corto plazo. Sin embargo, difícilmente llegará a sustituir al ser humano en su totalidad, sino que será un factor complementario en la toma de decisiones (Alonso y Carrio, 2019).

\section{Internet of things (Internet de las cosas)}

Es la capacidad que tienen los dispositivos físicos y digitales para interconectarse mediante el internet y compartir información entre sí. Ahora el internet no es tan solo una nube, sino que en conjunto con los dispositivos forman un todo. El internet de las cosas (IoT) permite a la banca utilizar los dispositivos móviles, pulseras, smartwatches, entre otros, como canales de comunicación para conocer mejor a sus clientes. Por lo tanto, permite a la banca ofrecer servicios financieros personalizados utilizando los propios dispositivos como fuente de información.

\section{Tecnología biométrica}

Sistemas de autenticación de identidad mediante el reconocimiento de personas a través de medios físicos como las huellas dactilares, lectura del iris, 
reconocimiento de voz o facial entre otros. Esta tecnología permite probar de forma automática e inequívoca la identidad de los clientes financieros. Facilita la identificación porque hace innecesaria la memorización de contraseñas para acceder a las plataformas, evita el uso de tarjetas magnéticas, previene el robo de contraseñas, entre otros.

La gran mayoría de los consumidores en el mundo $(93 \%)$ prefiere la biométrica sobre las contraseñas para validar un pago. El uso de la huella dactilar para acceder a determinados servicios móviles ha calado entre los consumidores convirtiéndolo en el método más aceptado (80\%) seguido por el reconocimiento facial $(56 \%)$ o de Iris (50\%). (Ditrendia, 2019, p.116)

Uso de canales digitales

\section{Banca móvil}

Es una aplicación donde los clientes pueden realizar operaciones financieras a través de un teléfono móvil de forma remota las 24 horas del día. La banca móvil reduce los costos de tiempo y dinero a favor de los clientes evitando el traslado a las oficinas del banco.

En general, la banca móvil brinda la posibilidad de derribar barreras importantes para la inclusión financiera de los pobres: asequibilidad y disponibilidad física. En comparación con los bancos tradicionales basados en sucursales, la banca móvil tiene la ventaja de que no incurre en el costo de implementación, maneja costos menores para las transacciones de bajo valor y proporciona un enlace con el nuevo nicho de mercado: las personas que no están bancarizadas. (Azabache, 2018, p.71)

\section{Banca por Internet}

Son operaciones financieras que realizan los clientes a través de una computadora o laptop, utilizando una conexión a internet en un espacio virtual World Wide Web (www).

Los servicios de banca por Internet son un elemento de importancia estratégica para las entidades bancarias, considerando su potencial para la reducción de costes e incremento de ingresos; por ello, los bancos buscan redirigir a sus clientes hacia sus servicios en Internet. (Bourgault, Egea, Guerrero, González, y Mangin, 2013, p.37)
Este servicio facilita la atención de los clientes en tiempo real sin la necesidad de ir físicamente al banco, lo que la convierte en una herramienta fundamental para la inclusión financiera.

\section{Infraestructura de telecomunicaciones}

\section{Internet en los hogares}

La marginalización de los peruanos en el uso de internet (los que no tienen acceso) representan un importante porcentaje: $47.46 \%$ (2018), 43.35\% (2019) y $38.92 \%$ (2020), lo que constituye un gran impedimento para el desarrollo de la digitalización de la banca. La penetración del internet en América Latina es de $68.66 \%$ (2018), 73.52\% (2019) y $78.78 \%$ (2020), lo que refleja el progreso en los últimos años (CAF, 2020b).

Tabla 2. Penetración del internet en América Latina (2018-2020).

\begin{tabular}{lccc}
\hline \multicolumn{1}{c}{ País } & $\mathbf{2 0 1 8}$ & $\mathbf{2 0 1 9}$ & $\mathbf{2 0 2 0}$ \\
\hline Argentina & $77.78 \%$ & $81.42 \%$ & $85.24 \%$ \\
Bolivia & $48.22 \%$ & $53.04 \%$ & $58.34 \%$ \\
Brasil & $74.22 \%$ & $81.64 \%$ & $89.80 \%$ \\
Chile & $82.33 \%$ & $82.33 \%$ & $82.33 \%$ \\
Colombia & $66.68 \%$ & $71.40 \%$ & $76.47 \%$ \\
Ecuador & $60.67 \%$ & $64.27 \%$ & $68.09 \%$ \\
México & $65.77 \%$ & $67.75 \%$ & $69.79 \%$ \\
Paraguay & $64.99 \%$ & $69.16 \%$ & $73.60 \%$ \\
Perú & $52.54 \%$ & $56.65 \%$ & $61.08 \%$ \\
Uruguay & $70.21 \%$ & $72.20 \%$ & $74.24 \%$ \\
América Latina & $68.66 \%$ & $73.52 \%$ & $78.78 \%$ \\
\hline
\end{tabular}

Fuente: Adaptado de Banco de Desarrollo de América Latina CAF, 2020b.

\section{Cobertura 5G en América Latina}

La tecnología $5 \mathrm{G}$ se refiere a la red móvil de quinta generación que aumenta la efectividad de la transmisión de datos y permite conectar múltiples dispositivos con una latencia mínima y gran ancho de banda. El $5 \mathrm{G}$ permite navegar hasta $10 \mathrm{GBps}$ (gigabytes por segundo), mucho más rápido que el 4G que conocemos. Asimismo, el tiempo de respuesta de la red (latencia) podrá reducirse a 5 milisegundos, lo que permitirá la comunicación en tiempo real.

En el 2025, América Latina alcanzará el $9 \%$ de las conexiones de internet móvil a través de la tecnología 5G, es decir, 62 millones de conexiones. Los países con pronósticos más alentadores en la región son Brasil 18\% y México 12\% (Figura 3), el 


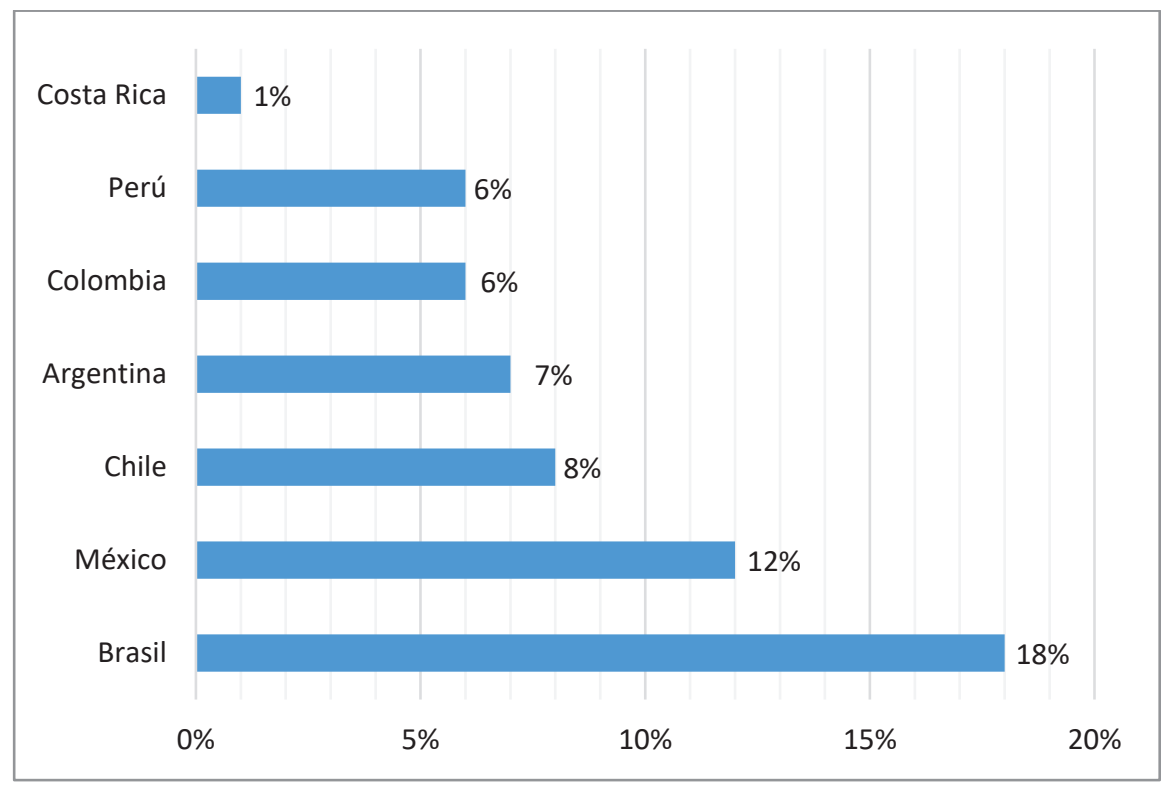

Figura 3. América Latina: Alcance estimado de la tecnología 5G por país al 2025. Fuente: Global System for Mobile Communications Association, 2020.

crecimiento será progresivo y seguirá el proceso de convivencia con la tecnología $4 \mathrm{G}$ durante los próximos años (Global System for Mobile Communications [GSMA], 2020).

El 21 de abril de 2021, el Ministerio de Transportes y Comunicaciones del Perú autorizó la implementación de la tecnología $5 G$ para servicios móviles, esta se realizará bajo el estándar NSA (sobre redes existentes) adjudicadas en licitaciones públicas. El gobierno acaba de publicar normas legales para la reasignación de $500 \mathrm{MHz}$ a las bandas de 1.7 $\mathrm{GHz}, 2.1 \mathrm{GHz}$ y $3.5 \mathrm{GHz}$. La implementación inicial $5 G$ en servicios móviles se iniciará en las provincias de Ica, Trujillo, Arequipa, Cañete, Huarochirí, Callao y Lima. Posteriormente, se irá ampliando en forma progresiva a otras zonas del país durante el 2022 (Ministerio de Transportes y Comunicaciones [MTC], 2021).

\section{Riesgos de la digitalización}

En América Latina, las organizaciones y usuarios de medios digitales han sufrido la suplantación de identidad, pérdidas de datos personales y la vulneración de la ciberseguridad al realizar transacciones bancarias. Según las entidades bancarias, los tipos de ataques más utilizados contra los clientes de servicios financieros fueron el phishing por correo electrónico (49.7\%), el phishing por mensaje de texto (24.2\%), el phishing por llamada de voz (24.8\%) y la infección con software malintencionado o malware (35.67\%) (Organización de Estados Americanos [OEA], 2018).

En relación con la materialización de incidentes (ataques exitosos) de seguridad digital (incluidos aspectos de seguridad de la información, ciberseguridad y prevención del fraude usando medios digitales) en las entidades bancarias en la región durante el 2017 , se resalta que el $65 \%$ de los Bancos grandes manifiestan que sí fueron víctimas de ataques exitosos, mientras que entre los Bancos medianos el porcentaje es del $43 \%$ y entre los pequeños, del 19\%. (OEA, 2018, p. 72) (Figura 4)

Los usuarios de servicios financieros que manifestaron no usar los servicios digitales para realizar sus operaciones bancarias proporcionaron las siguientes razones: desconfianza (59.26\%), falta de interés en los canales digitales (27.78\%), desconocimiento de tecnología digital $(11.11 \%)$ y falta de servicios digitales (9.26\%). A partir de estos resultados se puede concluir que es necesario generar confianza y seguridad en los usuarios para incentivar el uso de medios digitales (OEA, 2018).

\section{METODOLOGÍA}

"La inclusión financiera significa para las personas físicas y empresas, tener acceso a productos 
financieros útiles y asequibles" (Banco Mundial, 2018, párr. 1). A partir de esta definición solo se abordará la inclusión financiera con base en la población peruana en general, por lo que se incluyen todos los niveles socioeconómicos.

Esta investigación busca demostrar la relación significativa entre las variables banca digital e inclusión financiera en el Perú. Presenta un enfoque cuantitativo, porque se utilizarán herramientas estadísticas para analizar y explicar los resultados.
Es de tipo correlacional y diseño no experimental. La información será extraída de la base de datos STATISTA donde se analizará el periodo 20102019.

\section{RESULTADOS}

La variable independiente $(X)$ está representada por la penetración de la banca digital y la variable dependiente $(\mathrm{Y})$ está representada por las cuentas de depósitos bancarios que representan a la inclusión

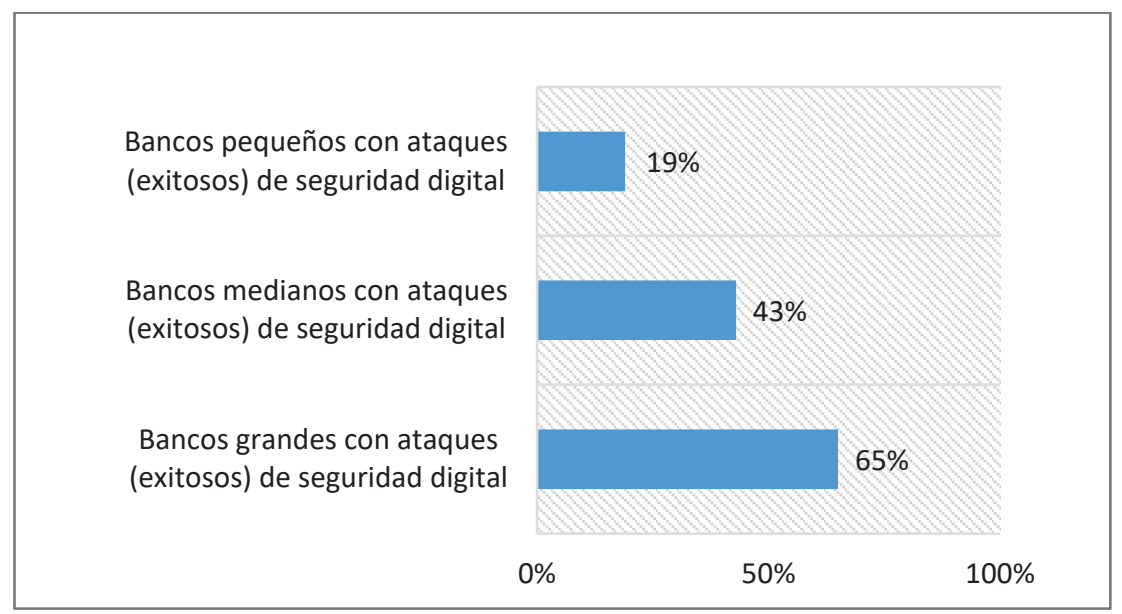

Figura 4. América Latina: Bancos víctimas de ataques de seguridad digital. Fuente: Organización de los Estados Americanos, 2018.

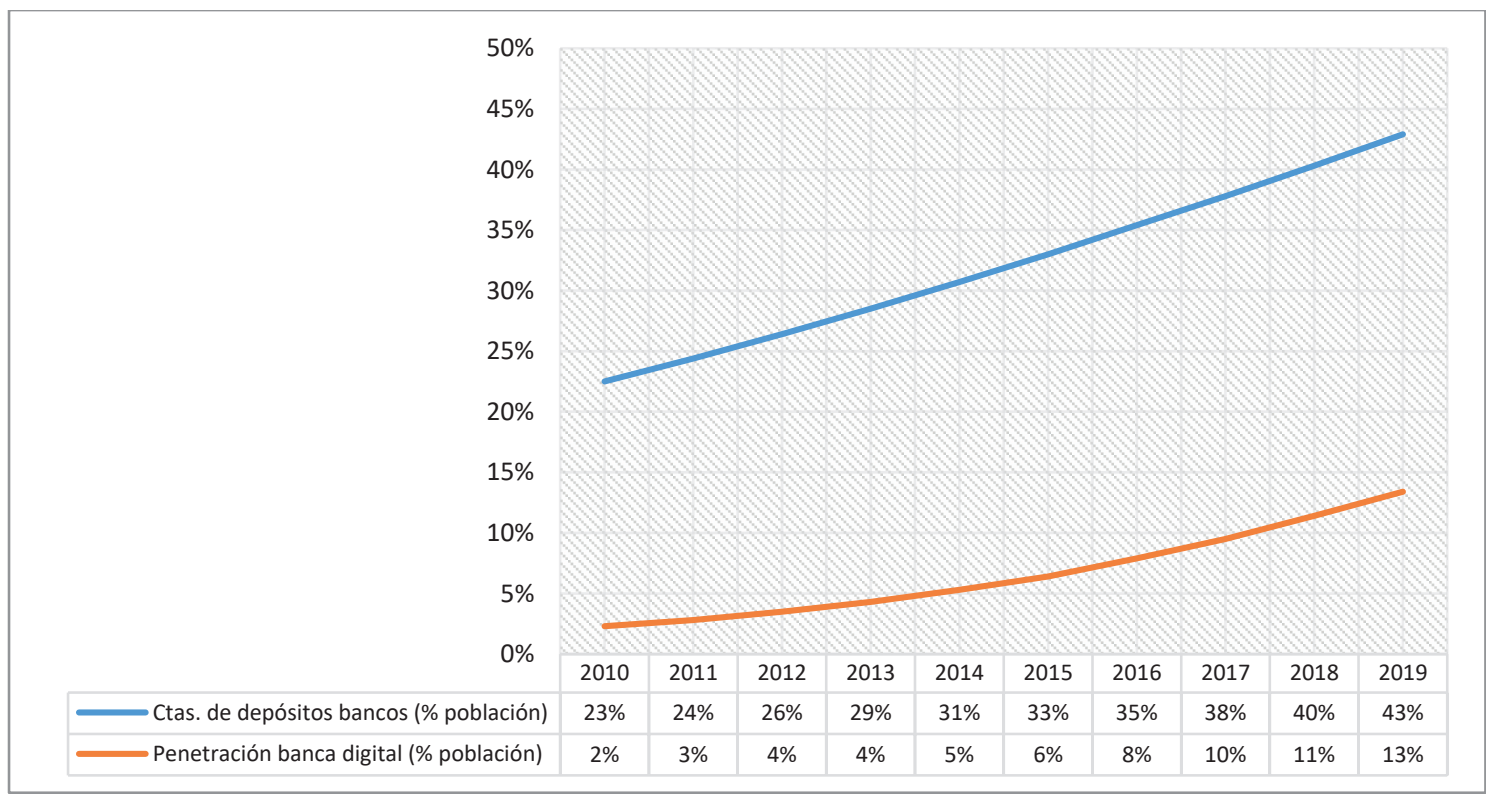

Figura 5. Perú: Cuentas de depósitos en bancos comerciales y penetración de la banca digital 2015-2019 con base en el porcentaje de la población.

Fuente: Adaptado de STATISTA, 2021. 
Tabla 3. Prueba de Normalidad.

\begin{tabular}{lcccccc}
\hline & \multicolumn{3}{c}{ Kolmogorov-Smirnov $^{\text {a }}$} & \multicolumn{3}{c}{ Shapiro-Wilk } \\
& Estadístico & gl & Sig. & Estadístico & gl & Sig. \\
\hline Penetración de la banca digital & 0.167 & 10 & $0.200^{*}$ & 0.931 & 10 & 0.455 \\
Inclusión financiera & 0.117 & 10 & $0.200^{*}$ & 0.961 & 10 & 0.795 \\
\hline
\end{tabular}

*. Esto es un límite inferior de la significación verdadera.

a. Corrección de significación Lilliefors.

Fuente: Elaboración propia a partir de SPSS-IBM.

financiera (Figura 5). La variable dependiente es la variable que se predice o calcula y la variable independiente proporciona la base para el cálculo, es decir, es la variable de predicción.

\section{Análisis estadístico}

Como la muestra de datos es $(n<30)$, se analizará la normalidad con Shapiro-Wilk. En la Tabla 3, se observa que las dos variables cuantitativas tienen un nivel de significancia (Sig. > 0.05), es decir, existe normalidad y se puede utilizar la correlación de Pearson.

Las hipótesis de investigación son:

$\mathrm{H}_{0=}$ La banca digital no se relaciona significativamente con la inclusión financiera.

$\mathrm{H}_{1=}$ La banca digital se relaciona significativamente con la inclusión financiera.

Para la interpretación de los datos se realizará un análisis de regresión y correlación lineal con el software SPSS-IBM.

Si el $p$-valor es $<0.05$ existe relación lineal y se acepta la hipótesis de investigación $\mathrm{H}_{1}$.

Las variables de investigación son:

X: Penetración de la banca digital con base en el porcentaje de la población (variable independiente)

Y: Cuentas de depósito en bancos con base en el porcentaje de la población - representando a la inclusión financiera (variable dependiente)

Dado el comportamiento de las variables independientes, se utilizará la técnica estadística de regresión lineal para predecir las variables dependientes.

Forma general de la ecuación de regresión (expresión matemática que define la relación entre dos variables).

$$
y=a+b x
$$

$y=\quad$ Variable dependiente

$a=\quad$ Ordenada y

$x=\quad$ Variable independiente

$b=\quad$ Pendiente de la recta

Se utilizará el análisis de correlación de Pearson como medida de dependencia que permite conocer el grado de asociación lineal entre dos variables cuantitativas $(\mathrm{X}, \mathrm{Y})$.

$\rho x \cdot y=\frac{\sigma x y}{\sigma x \sigma y}$

$\rho x . y=$ Coeficiente de correlación de Pearson

$\sigma x . y=$ Covarianza de $(\mathrm{X}, \mathrm{Y})$

$\sigma x=\quad$ Desviación estándar de $\mathrm{X}$

$\sigma y=\quad$ Desviación estándar de Y

Las variables penetración de la banca digital e inclusión financiera muestran el valor de correlación de Pearson positivo de 0.982 , muy cercano a 1 . El $p$-valor es $0.000<0.05$ (Tabla 4).

En el diagrama de dispersión se dibuja una nube de puntos en las coordenadas X, Y. Se observa una relación lineal, a medida que aumenta la variable $X$ también aumenta la variable Y (Figura 6).

El modelo resultante es: $Y=20.27+1.81 X$

\section{DISCUSIÓN}

El coeficiente de correlación de Pearson describe la intensidad de la relación de las variables, puede tomar cualquier valor de -1.00 a +1.00 , mientras el resultado obtenido se acerque a estos valores se puede decir que la correlación es perfecta.

Estadísticamente, se muestra una correlación de 0.982 , es decir, existe una fuerte correlación positiva cercana $a+1.00$. El $p$-valor es $0.000<0.05$, por 
Tabla 4. Correlación de Pearson.

\begin{tabular}{llcc}
\hline & & Penetración de la banca digital & Inclusión financiera \\
\hline \multirow{3}{*}{ Penetración de la banca digital } & Correlación de Pearson & 1 & $0.982^{* *}$ \\
& Sig. (bilateral) & & 0.000 \\
& $\mathrm{~N}$ & 20 & 10 \\
\hline \multirow{3}{*}{ Inclusión financiera } & Correlación de Pearson & $0.982^{\star *}$ & 1 \\
& Sig. & 0.000 & 10 \\
\hline
\end{tabular}

**. La correlación es significativa en el nivel 0.01 (bilateral).

Fuente: Elaboración propia a partir de SPSS-IBM.

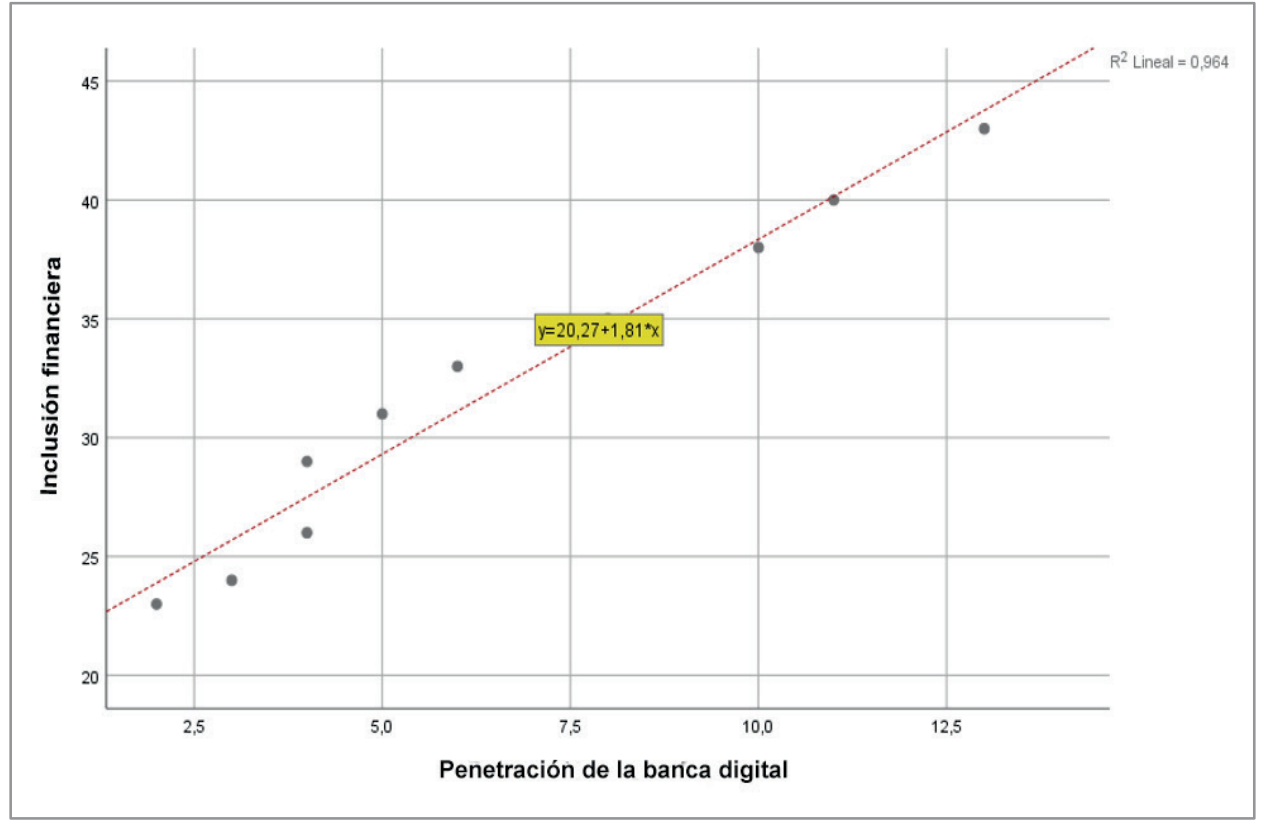

Figura 6. Diagrama de dispersión (correlación positiva). Fuente: Elaboración propia a partir de SPSS-IBM.

lo tanto, se acepta la $\mathrm{H}_{1}$ : La banca digital se relaciona significativamente con la inclusión financiera, porque las variables están altamente correlacionadas. La prueba estadística se realizó al $5 \%$ de error o 95\% de confianza. El modelo gráfico indica que, por cada punto porcentual que incrementa la banca digital, la inclusión financiera aumenta en 1.81 puntos porcentuales.

El diagrama de dispersión indica que, a medida que se incrementan las operaciones por la banca digital, también se incrementa la inclusión financiera, recta con pendiente positiva.

\section{CONCLUSIONES}

Al haberse comprobado la hipótesis de estudio se puede afirmar que la banca digital se relaciona significativamente con la inclusión financiera porque ambas variables están altamente correlacionadas. La banca digital se convierte en una alternativa estratégica para la inclusión financiera en el Perú, al aplicar tecnologías innovadoras y software para optimizar los servicios financieros y llegar a las poblaciones más alejadas en el territorio nacional de una forma participativa y al menor costo.

La banca digital le permite al sistema financiero expandir sus servicios mediante plataformas de internet o teléfonos móviles, pues permite abrir cuentas de ahorro, adquirir seguros, solicitar préstamos o tarjetas de crédito, realizar inversiones en fondos mutuos, entre otros. Sin embargo, tal como señala la OEA (2018), los incidentes de seguridad digital experimentados por los usuarios del sistema bancario en América Latina son el phishing por correo electrónico (49.7\%), el phishing por mensaje de texto $(24.2 \%)$, el phishing por llamada de voz 
(24.8\%) y la infección con software malintencionado o malware (35.67\%).

En el Perú la penetración de internet fue de 53\% (2018), 57\% (2019) y 61\% (2020), debido a limitaciones de conectividad. Como consecuencia de esto, se intensifica el manejo de dinero en efectivo y sus desventajas como: peligro de robos, uso inapropiado, falsificaciones, fraudes, lavado de activos, entre otros.

La banca tradicional se enfrenta a grandes desafíos como la transformación digital que implica inversión en tecnología, cambio de cultura, nuevos modelos de negocio, recursos humanos entre otros, con la finalidad de adaptarse a los actuales requerimientos del sector y entorno.

\section{REFERENCIAS BIBLIOGRÁFICAS}

[1] Alonso, I., y Carrio, A. (2019). La gestión de la información en banca: de las finanzas del comportamiento a la inteligencia artificial. Papeles de Economía Española (162), 148161. Recuperado de https://www.funcas.es/ revista/la-gestion-de-la-informacion-en-bancade-las-finanzas-del-comportamiento-a-lainteligencia-artificial-febrero-2020/

[2] Asobancaria (2017). La banca digital: estrategia clave para impulsar la inclusión financiera. Semana Económica. Recuperado de http://www.asobancaria.com/ semanseconomicas/1074-a.pdf

[3] Azabache, C. (2018). La banca móvil como alternativa para la inclusión social en áreas rurales. Review of Global Management, 3(2), 67-81. Recuperado de https://dx.doi. org/10.19083/rgm.v3i2.779

[4] Banco Mundial. (2018). Inclusión financiera. Recuperado de https://www.bancomundial.org/ es/topic/financialinclusion/overview

[5] Bourgault, N., Egea, J., Guerrero, M., González, M., y Mangin, J. (2013). Modelización de la confianza y seguridad en la adopción de la banca por internet. Revista Portuguesa de Marketing, 16(30), 35-54. Recuperado de https://search.proquest.com/scholarly-journals/ modelización-de-la-confianza-y-seguridad-en/ docview $/ 1462485767 /$ se- 2 ? accountid=12268

[6] Bueno, E., Longo, M., y Morcillo, P. (2017). La innovación del modelo de negocio bancario: El reto de la banca digital. Revista de la Asociación Española de Contabilidad y Administración de
Empresas, 5-8. Recuperado de https://aeca. es/wp-content/uploads/2019/10/120.pdf

[7] Comisión Económica para América Latina y el Caribe. (2020). Fortalecimiento de la inclusión y capacidades financieras en el ámbito rural. Recuperado de https://repositorio.cepal.org/ bitstream/handle/11362/45115/4/S2000323_ es.pdf

[8] Corporacion Andina de Fomento. (2020a). Las oportunidades de la digitalización en América Latina fente al Covid-19. Recuperado de https:// scioteca.caf.com/handle/123456789/1541

[9] Corporacion Andina de Fomento. (2020b). El estado de la digitalización de América Latina frente a la pandemia del COVID-19. Recuperado de https://scioteca.caf.com/ handle/123456789/1540

[10] Ditrendia. (2019). Mobile en España y en el Mundo 2019. Recuperado de https://ditrendia. es/informe-mobile-espana-mundo-2019/

[11] Federación Latinoamericana de Bancos. (2018). El blockchain y los smart contracts en la banca y el sector financiero. Recuperado de https://felaban.s3-us-west-2.amazonaws.com/ noticias/Noticia-2018-07-18.pdf

[12] Fondo Monetario Internacional. (2020). Financial Access Survey. Recuperado de https://data.imf.org/?sk=E5DCAB7E-A5CA4892-A6EA-598B5463A34C

[13] Global System for Mobile Communications. (2020). The mobile economy Latin America 2020. Recuperado de https://www.gsma.com/ mobileeconomy/wp-content/uploads/2020/12/ GSMA_MobileEconomy2020_LATAM_Eng. pdf

[14] Grupo Consultivo de Ayuda a los Pobres. (2015). Inclusión financiera digital: Implicancias para clientes, reguladores, supervisores y organismos normativos. Recuperado de https:// www.cgap.org/sites/default/files/Brief-DigitalFinancial-Inclusion-Feb-2015-Spanish.pdf

[15] Ministerio de Transportes y Comunicaciones. (2021). MTC autoriza el despliegue de tecnología $5 G$ para servicios móviles. Recuperado de https://www.gob.pe/institucion/ $\mathrm{mtc} /$ noticias/482361-mtc-autoriza-eldespliegue-de-tecnologia-5g-para-serviciosmoviles

[16] Orazi, S., Martinez, L., y Vigier, H. (2019). La inclusión financiera en América Latina y Europa. Ensayos de Economía, 29(55), 181- 
204. Recuperado de https://doi.org/10.15446/ ede.v29n55.79425

[17] Organización de Estados Americanos. (2018). Estado de la Ciberseguridad en el Sector Bancario en América Latina y el Caribe. Recuperado de https://www.oas.org/es/sms/ cicte/sectorbancariospa.pdf

[18] Organización de las Naciones Unidas. (2020). Technology and Innovation Report. Recuperado de https://unctad.org/es/node/32191

[19] Revuelta, M. (2018). "Big Data": crisis y nuevos plantaeamientos en los flujos de comunicación de la cuarta revolución industrial. Área Abierta, 18(2), 309-324. Recuperado de: http://dx.doi. org/10.5209/ARAB.59521
[20] Serrano-Cobos, J. (2016). Tendencias tecnológicas en internet: Hacia un cambio de paradigma. El Profesional de la Información, 25(6), 843-850. Recuperado de http://dx.doi. org/10.3145/epi.2016.nov.01

[21] STATISTA. (2021). Digital Payments. Recuperado de https://www.statista.com/ outlook/dmo/fintech/digital-payments/southamerica 


\section{Digital Banking: Technological Innovation in Financial Inclusion in Peru}

Allan Herminio Vargas Garcia ${ }^{1}$

\begin{abstract}
Technology-driven digital transformation is impacting the financial sector with the entry of new business models such as fintech and bigtech that successfully compete by offering financial products with disruptive potential. Digital banking is the result of the sum of traditional banking and the internet that offers banking services through a website or mobile application. The integration of financial services into digital ecosystems represents a challenge for traditional banking that needs to evolve to face the changing environment. Digital banking plays an important role in financial inclusion, as it allows access to innovative services to more Peruvians and boosts economic growth. However, a high level of digital fraud also exists, aimed at misappropriating money or blackmailing financial users by means of phishing and the use of malware. Pearson's correlation coefficient, useful for linear relationships, was used to evaluate the variables "digital banking" and "financial inclusion", and SPSS-IBM software was used to tabulate the results.
\end{abstract}

Keywords: innovation; technology; inclusion; finance; banking.

\section{INTRODUCTION}

The significance of technologies used by digital banking is studied in this research, as they offer an excellent alternative for the integration of the socially-excluded population by providing access to and use of financial services (financial inclusion). Such technologies, starting from data traffic on cell phones, interbank transactions and digital accounts, can be used as tools to improve customer service efficiency.

Financial inclusion is a multidimensional concept related to the supply and demand aspects of financial products or services, whose dimensions are access, use, quality and impact on the financial wellbeing of families and companies. According to the Economic Commission for Latin America and the Caribbean (ECLAC), financial inclusion is a factor that drives 7 of the 17 sustainable development goals: "(SDG1) No poverty, (SDG2) Zero hunger, (SDG3) Good health and well-being, (SDG5) Gender equality, (SDG8) Decent work and economic growth, (SDG9) Industry, innovation and infrastructure, and (SDG10) Reduced inequalities" (ECLAC, 2020, p.14), making this research particularly relevant. The objective of this study is to determine to what extent digital banking is related to financial inclusion during the period 2010-2019, while the general hypothesis seeks to verify that digital banking is significantly related to financial inclusion in Peru during the period 2010-2019.

This research contributes to expanding knowledge regarding the change that technological innovation is bringing about in the use of and access to financial services, which suggests that the future of banking and the development of financial inclusion is digital.

\section{Digitalization in Latin America}

In terms of digital development, Latin America ranks at a medium level with an index of 49.92 (on a scale of 0 to 100), higher than the African region (35.05) and Asia Pacific (49.16). However, it

1 Master in Administration from Universidad del Pacífico. Currently working as professor at the School of Administrative Sciences of Universidad Nacional Mayor de San Marcos. (Lima, Peru). ORCID: https://orcid.org/0000-0003-2092-8706

E-mail: avargasg@unmsm.edu.pe 
falls behind Eastern Europe (52.90), Western Europe (71.06) and North America (80.85). As part of the developing world, Latin America shows a moderate growth rate of digitalization compared to other regions of the developed world (Corporación Andina de Fomento [CAF], 2020a).

The Frontier Technologies Readiness Index prepared by the United Nations [ONU] (2020) presented the results obtained by 158 countries based on a scale from 0 to 1 , placing them within one of four 25 -percentile score groups. The values considered were: low, lower-middle, upper-middle, and high. Table 1 shows the position obtained by the countries representing Latin America.

Table 1. Latin America's Technologies Index.

\begin{tabular}{lccc}
\hline \multicolumn{1}{c}{ Country } & $\begin{array}{c}\text { World } \\
\text { Ranking }\end{array}$ & Score & Score Group \\
\hline Brazil & 41 & 0.65 & Upper-middle \\
Chile & 49 & 0.57 & Upper-middle \\
Mexico & 57 & 0.54 & Upper-middle \\
Argentina & 65 & 0.49 & Upper-middle \\
Uruguay & 68 & 0.47 & Upper-middle \\
Colombia & 78 & 0.44 & Upper-middle \\
Peru & 89 & 0.36 & Lower-middle \\
Ecuador & 90 & 0.34 & Lower-middle \\
Paraguay & 102 & 0.29 & Lower-middle \\
Bolivia & 116 & 0.24 & Lower-middle \\
\hline
\end{tabular}

Source: Adapted from the United Nations, 2020.

\section{Financial Inclusion}

Financial inclusion favors economic growth, focusing on the vulnerable population, as it provides the opportunity to save, invest, borrow and obtain credit and insurance through the use of and access to financial services. Use of and access to financial services is among the economic and social differences between high-income countries in Europe and low-income countries in Latin America (Orazi, Martínez, \& Vigier, 2019).

\section{Similarly,}

Being able to have access to a transaction account is a first step toward broader financial inclusion since a transaction account allows people to store money, and send and receive payments. A transaction account serves as a gateway to other financial services, which is why ensuring that people worldwide can have access to a transaction account is the focus of the World Bank
Group's Universal Financial Access 2020 initiative. (Banco Mundial, 2018, para. 2)

In 2019, regarding the number of deposit accounts in commercial banks, Mexico had 98 million 623 thousand, Colombia had 75 million 728 thousand; Argentina had 67 million 579 thousand; Chile had 44 million 994 thousand; Peru had 42 million 662 thousand; and finally Bolivia had 6 million 256 thousand (Figure 1).

Concepts such as savings or access to credit are important aspects of financial inclusion. In 2019, regarding loan accounts in commercial banks, Colombia had 24 million 463 thousand; Argentina had 18 million 997 thousand; Chile had 15 million 675 thousand; Peru had 5 million 923 thousand; and finally Bolivia had 692 thousand (Figure 2).

Digital financial inclusion represents an opportunity for neglected low-income families to integrate into the banking sector and access digital financial services through the internet.

"Digital financial inclusion" can be defined as digital access to and use of formal financial services by excluded and underserved populations. Such services should be suited to the customers' needs and delivered responsibly, at a cost both affordable to customers and sustainable for providers. (Grupo Consultivo de Ayuda a los Pobres [CGAP], 2015, p. 1)

\section{Digital Banking}

New disruptive technologies have prompted traditional banking to be more competitive and a) be fast and accurate in risk assessment; $b$ ) ensure the security and protection of customer data; c) exploit big data intelligence to generate value from users; and d) offer products and services according to the reality and need of digital customers (Bueno, Longo, \& Morcillo, 2017).

A definition of digital banking is presented below.

(...) el mundo digital ha cambiado la dinámica tradicional de la banca, ofreciendo productos enfocados en el consumidor y sus necesidades. Se trata de un consumidor que demanda transacciones en tiempo real, seguras $y$ eficientes, $y$ al que las nuevas tecnologías lo empoderan para: a) administrar sus productos a través de plataformas a las que pueden acceder desde cualquier lugar y en cualquier momento; b) conocer de primera mano la oferta de productos $y$ 


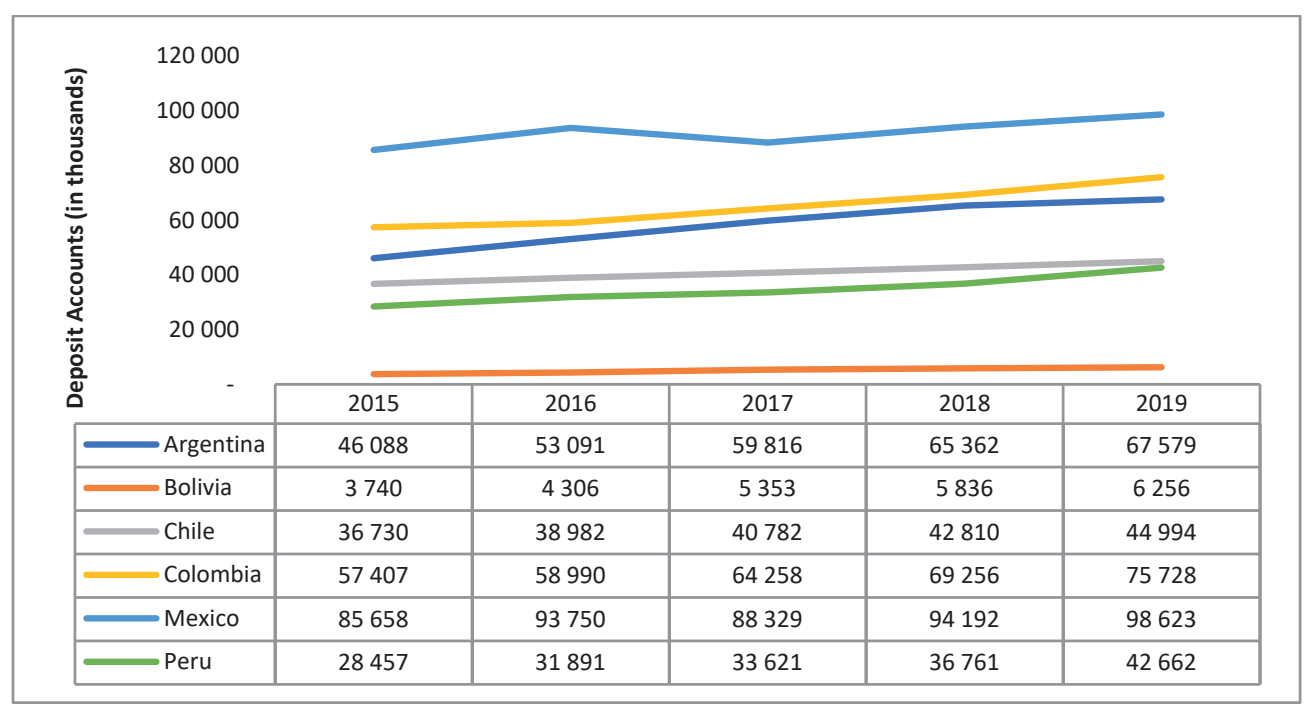

Figure 1. Latin America: Number of deposit accounts in commercial bank branches from 2015-2019 (in thousands). Source: Adapted from International Monetary Fund, 2020.

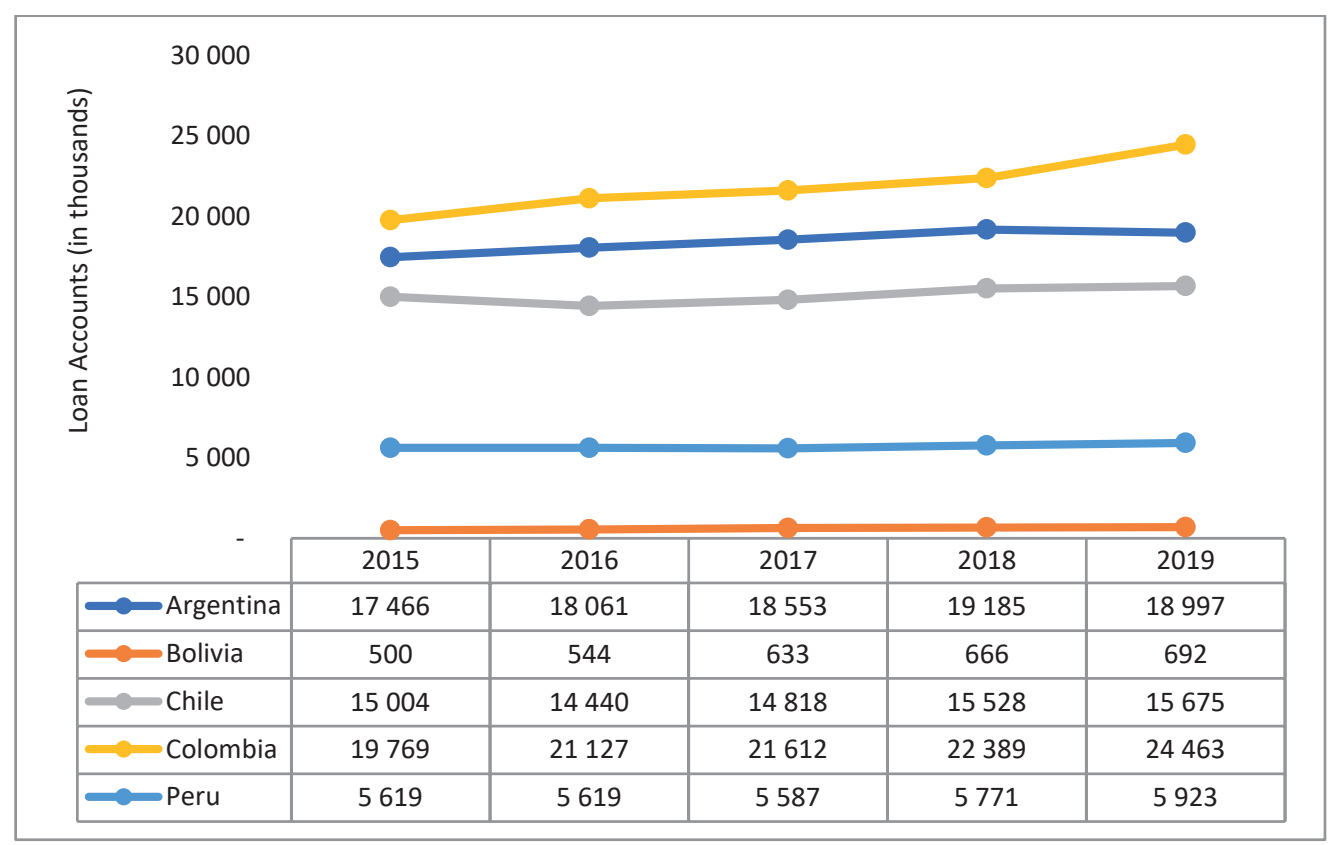

Figure 2. Latin America: Number of loan accounts in commercial bank branches from 2015-2019 (in thousands). Source: Adapted from International Monetary Fund, 2020.

servicios; c) comparar las opciones que le ofrece el mercado $y$ d) emitir opiniones y defender sus derechos de manera más efectiva. Otra faceta de las nuevas tecnologías consiste en el impacto potencial que tienen para impulsar la inclusión financiera, particularmente en sectores de bajos ingresos [the digital world has changed the traditional dynamics of banking, offering products focused on the consumer and their needs. These consumers demand real-time, secure and efficient transactions, and new technologies empower them to: a) manage their products through platforms they can access from anywhere and at any time; b) learn first-hand about the range of products and services available; c) compare the options offered by the market; and d) express their opinions and defend their rights more 
effectively. A further feature of the new technologies is their potential to boost financial inclusion, particularly in low-income sectors]. (Asobancaria, 2017, p. 2)

\section{Digital Banking Technologies}

\section{Big data}

Big data is the massive collection and management of information that makes possible the storage, processing and analysis of digital information. It allows banks to make more effective decisions, as, based on a better knowledge of their customers, they are able to offer financial products and services designed according to their needs. It improves credit approval, risk management, investment banking and cross-selling processes. It also erases the barriers of information asymmetry.

El término Big Data no sólo hace referencia al volumen de datos, sino a las tecnologías asociadas a la captación, administración y visibilidad de los mismos; y no sólo se tiene en cuenta el volumen sino la variedad de los mismos y la velocidad de acceso y procesamiento. [The term Big Data refers not only to the volume of data, but also to the technologies associated with the collection, management and visibility of the data; not only volume but also the variety of the data and the speed of access and processing are taken into account]. (Revuelta, 2018, p.312)

\section{Blockchain}

A blockchain is a distributed database shared by a network that uses encrypted algorithms that makes information difficult to hack or steal. In banking, in the near future, blockchain will facilitate the secure transmission of data and capital using encrypted codes. It will also make it possible to provide customers with a secure identity on the web. Moreover, traditional contracts will be replaced by smart contracts, thus reducing the risk of transactions.

En cuanto al sector bancario y financiero, si bien es cierto que el uso de esta tecnología aún es incipiente, algunos bancos han venido trabajando de manera importante en la utilización del blockchain en el desarrollo de sus productos, viendo en su utilización enormes posibilidades para la reducción de costos, mayor eficiencia y agilidad en las transacciones. [As for the banking and financial sector, while the use of this technology is still incipient, certain banks have been actively using blockchain for product development, as they see in its use tremendous potential for cost reduction, greater efficiency and agility in transactions]. (Federación Latinoamericana de Bancos [FELABAN], 2018, p. 1)

\section{Cloud Computing}

Cloud computing is the technology that, via the Internet, enables data storage and facilitates access to platforms, applications and services in a virtual space. Clouds (servers located in the network) are where workloads are executed. Data storage and services in the cloud ensure a high-speed processing of transactions resulting in cost savings for both the financial client and the bank, creating scalability and agility in the banking business. It is also friendly with other technologies such as artificial intelligence and big data.

\section{Artificial Intelligence (AI)}

$\mathrm{Al}$ is a branch of computer science that deals with the development of systems to perform complex tasks as human intelligence would. Al has an impact on banking activities such as risk management, credit scoring, credit card fraud, cash transactions and personalized customer support (app chatbot). "Un chatbot es un software que interactúa con el usuario mediante una interfaz de conversación, diseñado para simular una conversación inteligente sin un ser humano presente [A chatbot is a software that interacts with the user via a conversational interface and is designed to simulate a conversation intelligently performed without a human being present]" (Serrano-Cobos, 2016, p. 845).

Al technology has become an essential tool for financial investment markets, which is why it will be widely used in the short term. However, it will hardly replace the human being in its entirety but rather it will be an additional factor in decision making (Alonso \& Carrio, 2019).

\section{Internet of things (IoT)}

It is the ability of physical and digital devices to interconnect through the Internet and share information with each other. The internet is no longer just a cloud, but together with the devices they form a whole. The loT enables banks to use mobile devices, wristbands, smartwatches, among others, as communication channels to better know their customers. Thus, it allows banks to offer personalized financial services using the devices as a source of information. 


\section{Biometric Technology}

Biometric technology systems are identity authentication systems that recognize people via physical means such as fingerprints, iris reading, voice or facial recognition, or other means. This technology enables automatic and unequivocal verification of the identity of financial customers. Identification is rendered more easily as it is unnecessary to memorize passwords to access platforms, it avoids the use of magnetic cards, and it prevents password theft.

La gran mayoría de los consumidores en el mundo (93\%) prefiere la biométrica sobre las contraseñas para validar un pago. El uso de la huella dactilar para acceder a determinados servicios móviles ha calado entre los consumidores convirtiéndolo en el método más aceptado (80\%) seguido por el reconocimiento facial (56\%) o de Iris (50\%) [Most consumers in the world (93\%) prefer biometrics over passwords to validate a payment. The use of fingerprint to access certain mobile services gained traction among consumers making it the most accepted method $(80 \%)$ followed by facial $(56 \%)$ or iris $(50 \%)$ recognition]. (Distrendia, 2019, p. 116)

\section{Use of Digital Channels}

\section{Mobile Banking}

Mobile banking is a service provided through an application where customers can perform remote financial transactions via a cell phone 24 hours a day. Mobile banking reduces time and money costs for clients, as they no longer have to go to the bank.

En general, la banca móvil brinda la posibilidad de derribar barreras importantes para la inclusión financiera de los pobres: asequibilidad y disponibilidad física. En comparación con los bancos tradicionales basados en sucursales, la banca móvil tiene la ventaja de que no incurre en el costo de implementación, maneja costos menores para las transacciones de bajo valor y proporciona un enlace con el nuevo nicho de mercado: las personas que no están bancarizadas [All in all, mobile banking has the potential to break down key barriers to financial inclusion for the disadvantaged: affordability and physical availability. Compared to traditional branch-based banks, mobile banking has the advantage no implementation cost, lowering fees for low-value transactions, and providing a bridge to the new niche market: the unbanked]. (Azabache, 2018, p. 71)

\section{Internet Banking}

Internet banking are financial transactions performed by customers via a computer or laptop, using an Internet connection on a virtual World Wide Web (www) space.

Los servicios de banca por Internet son un elemento de importancia estratégica para las entidades bancarias, considerando su potencial para la reducción de costes e incremento de ingresos; por ello, los bancos buscan redirigir a sus clientes hacia sus servicios en Internet [Internet banking services are a strategically important element for banking entities, considering their potential for cost reduction and revenue increase; therefore, banks seek to redirect their customers to their Internet services]. (Bourgault, Egea, Guerrero, González, \& Mangin, 2013, p. 37)

It enables real-time customer service without the need to physically go to the bank, which makes it a fundamental tool for financial inclusion.

\section{Telecommunications Infrastructure}

\section{Home internet}

Peruvians' marginalization regarding internet use (those who do not have access) represent a significant percentage: $47.46 \%$ (2018), $43.35 \%$ (2019) and $38.92 \%$ (2020), which is a major barrier preventing banking digitalization. Internet penetration in Latin America is $68.66 \%$ (2018), $73.52 \%$ (2019) and $78.78 \%(2020)$, which reflects progress in recent years (CAF, 2020b).

Table 2. Internet Penetration in Latin America (20182020).

\begin{tabular}{lccc}
\hline \multicolumn{1}{c}{ Country } & $\mathbf{2 0 1 8}$ & $\mathbf{2 0 1 9}$ & $\mathbf{2 0 2 0}$ \\
\hline Argentina & $77.78 \%$ & $81.42 \%$ & $85.24 \%$ \\
Bolivia & $48.22 \%$ & $53.04 \%$ & $58.34 \%$ \\
Brazil & $74.22 \%$ & $81.64 \%$ & $89.80 \%$ \\
Chile & $82.33 \%$ & $82.33 \%$ & $82.33 \%$ \\
Colombia & $66.68 \%$ & $71.40 \%$ & $76.47 \%$ \\
Ecuador & $60.67 \%$ & $64.27 \%$ & $68.09 \%$ \\
Mexico & $65.77 \%$ & $67.75 \%$ & $69.79 \%$ \\
Paraguay & $64.99 \%$ & $69.16 \%$ & $73.60 \%$ \\
Peru & $52.54 \%$ & $56.65 \%$ & $61.08 \%$ \\
Uruguay & $70.21 \%$ & $72.20 \%$ & $74.24 \%$ \\
Latin America & $68.66 \%$ & $73.52 \%$ & $78.78 \%$ \\
\hline
\end{tabular}

Source: Adapted from Banco de Desarrollo de América Latina CAF, 2020b. 


\section{G in Latin America}

$5 \mathrm{G}$ technology refers to the fifth generation mobile network that increases data transmission efficiency and allows multiple devices to be connected with minimal latency and high bandwidth. 5G allows browsing up to $10 \mathrm{GBps}$ (gigabytes per second), much faster than the $4 \mathrm{G}$ technology. In addition, network response time (latency) will be reduced to 5 milliseconds, enabling real-time communication.

By 2025, $5 \mathrm{G}$ penetration in Latin America is expected to reach $9 \%$, i.e. 62 million connections. The countries with the most encouraging forecasts in the region are Brazil with $18 \%$ and Mexico with $12 \%$ (Figure 3 ). $5 \mathrm{G}$ growth will be progressive and will coexist with $4 G$ technology over the next few years (Global System for Mobile Communications [GSMA], 2020).

On April 21, 2021, the Peruvian Ministry of Transport and Communications authorized the implementation of $5 \mathrm{G}$ technology for mobile services, to be implemented under the NSA standard over existing networks awarded in public tenders. The government has just published legal norms for the reallocation of $500 \mathrm{MHz}$ to the $1.7 \mathrm{GHz}, 2.1 \mathrm{GHz}$ and 3.5 $\mathrm{GHz}$ bands. Deployment of $5 \mathrm{G}$ in mobile services will start in the provinces of Ica, Trujillo, Arequipa, Cañete, Huarochirí, Callao and Lima. Subsequently, it will be progressively extended to other areas of the country during 2022 (Ministerio de Transportes y Comunicaciones [MTC], 2021).

\section{Risks of Digitalization}

In Latin America, organizations and users of digital technologies have experienced identity theft, loss of personal data and cybersecurity breaches in banking transactions. According to banking entities, the most frequently used types of attacks against financial services customers were email phishing $(49.7 \%)$, text message phishing $(24.2 \%)$, phone call phishing $(24.8 \%)$ and infection with malicious software or malware (35.67\%) (Organización de los Estados Americanos [OEA], 2018).

In relation to the materialization of digital security incidents (successful attacks) (including aspects of information security, cybersecurity and fraud prevention using digital media) in banking entities in the region during 2017, it is highlighted that $65 \%$ of the large banks state that they were victims of successful attacks, while among the medium banks the percentage is $43 \%$ and among the small banks, $19 \%$. (OEA, 2018, p. 72) (Figure 4)

Users of financial services who stated that they did not use digital services to perform their banking operations provided the following reasons: distrust $(59.26 \%)$, lack of interest in digital channels $(27.78 \%)$, lack of knowledge of digital technology $(11.11 \%)$ and lack of digital services $(9.26 \%)$. From these results, it can be concluded that it is necessary to build trust and confidence in users to encourage the use of digital media (OEA, 2018).

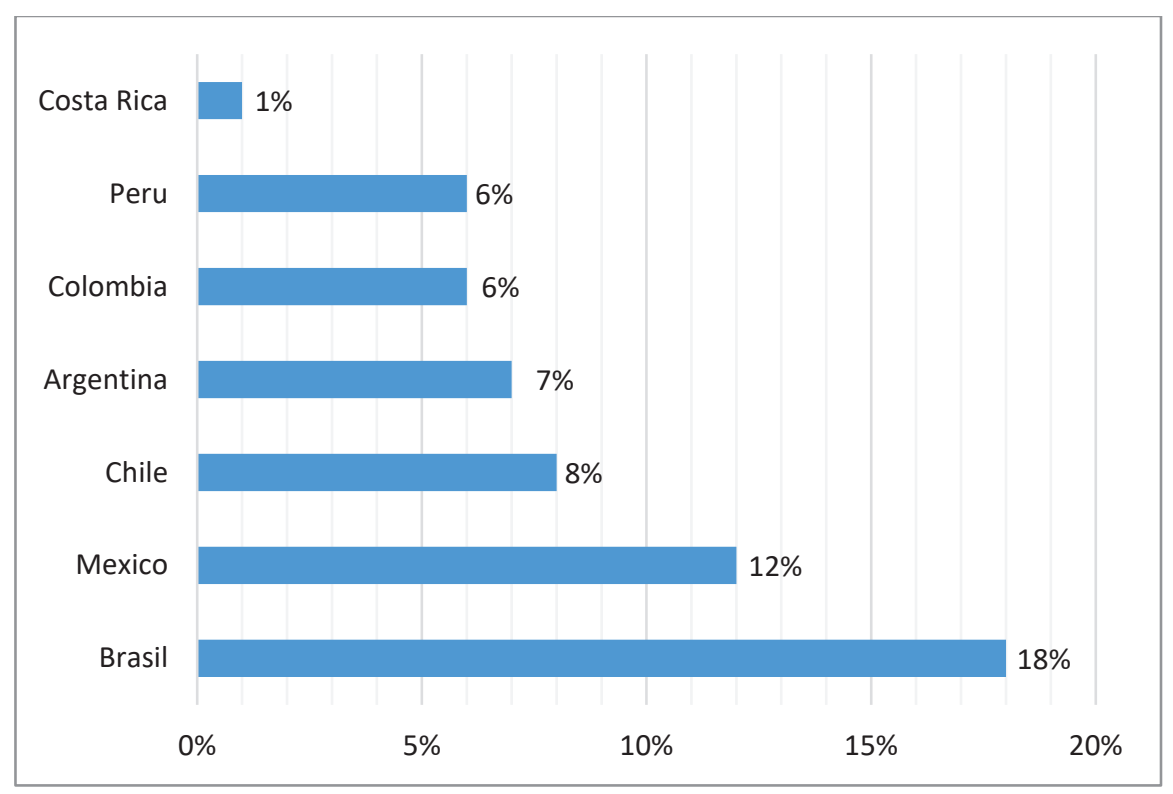

Figure 3. Latin America: Expected $5 G$ penetration by county by 2025.

Source: Global System for Mobile Communications Association, 2020. 


\section{METHODOLOGY}

"Financial inclusion means that individuals and businesses have access to useful and affordable financial products and services that meet their needs" (Banco Mundial, 2018, para. 1). Based on this definition, financial inclusion will only be addressed based on the Peruvian population in general, thus including all socioeconomic levels.

This research aims to demonstrate the significant relationship between the variables digital banking and financial inclusion in Peru. It has a quantitative approach, because statistical tools will be used to analyze and explain the results. It has a correlational and non-experimental design. Data for the period 2010-2019 will be obtained from the STATISTA database.

\section{RESULTS}

The independent variable $(X)$ is represented by digital banking penetration and the dependent variable $(\mathrm{Y})$ is represented by bank deposit accounts representing financial inclusion (Figure 5). The dependent variable is predicted or calculated, and the

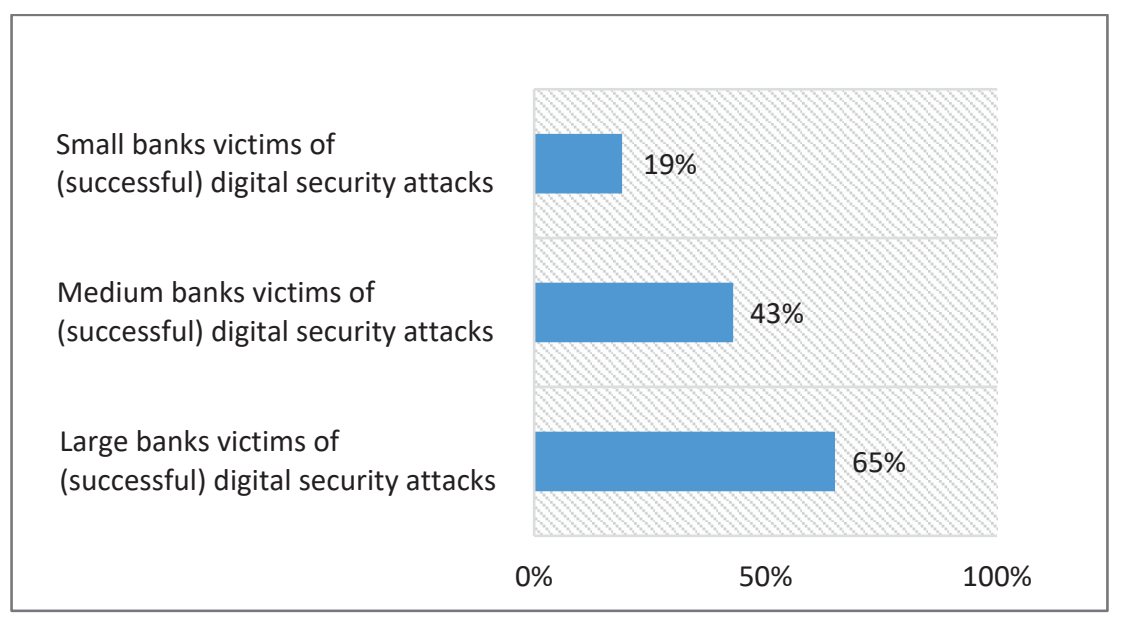

Figure 4. Latin America: Banks victims of digital security attacks. Source: Organización de los Estados Americanos,2018.

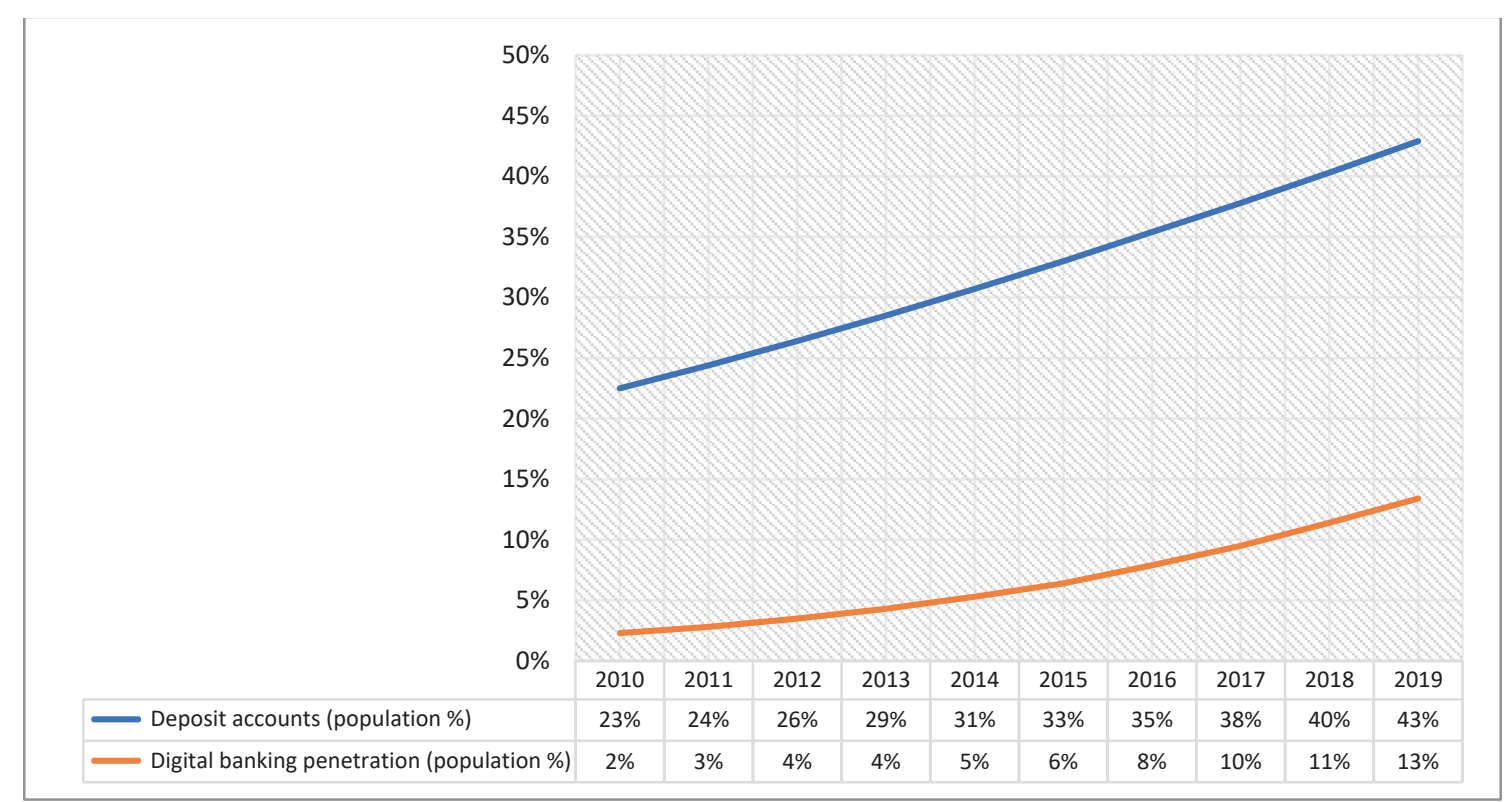

Figure 5. Peru: Commercial bank deposit accounts and digital banking penetration 2015-2019 based on population percentage.

Source: Adapted from STATISTA, 2021. 
independent variable provides the basis for the calculation, i.e., it is the predictor variable.

\section{Statistical Analysis}

As the data sample is $(n<30)$, normality will be analyzed with Shapiro-Wilk. Table 3 shows that the two quantitative variables have a significance level (Sig. > 0.05), thus, normality exists and Pearson's correlation can be used.

The research hypotheses are:

$\mathrm{H}_{0}=$ Digital banking is not significantly related to financial inclusion.

$\mathrm{H}_{1}=$ Digital banking is significantly related to financial inclusion.

A regression and linear correlation analysis will be performed using SPSS-IBM software to interpret the data.

If the $p$-value is $<0.05$ there is a linear relationship and the research hypothesis $\mathrm{H}_{1}$ is accepted.

The research variables are:

$\mathrm{X}$ : Digital banking penetration based on percentage of population (independent variable).

Y: Deposit accounts in banks based on the percentage of the population - representing financial inclusion (dependent variable).

A statistical technique of linear regression will be used to predict the dependent variables based on the behavior of the independent variables.

General form of the regression equation (mathematical expression that defines the relationship between two variables).

$y=a+b x$

$y=\quad$ Dependent variable $a=\quad$ Ordinate $\mathrm{y}$

$x=\quad$ Independent variable

$b=\quad$ Slope of the line

Pearson's correlation analysis will be used as a measure of dependence that provides information on the degree of linear association between two quantitative variables $(\mathrm{X}, \mathrm{Y})$.

$\rho x . y=\frac{\sigma x y}{\sigma x \sigma y}$

$\rho x \cdot y=$ Pearson's correlation coefficient

$\sigma x \cdot y=$ Covariance of $(X, Y)$

$\sigma x .=$ Standard deviation of $\mathrm{X}$

$\sigma . y=\quad$ Standard deviation of $Y$

Digital banking penetration and financial inclusion variables present the positive Pearson's correlation value of 0.982 , very close to 1 . The $p$-value is 0.000 $<0.05$ (Table 4).

The scatter plot depicts a cloud of points in the coordinates $\mathrm{X}, \mathrm{Y}$. A linear relationship is apparent, as the $X$ variable increases so does the $Y$ variable (Figure 6).

The resulting model is: $\mathrm{Y}=20.27+1.81 \mathrm{X}$

\section{DISCUSSION}

Pearson's correlation coefficient describes the intensity of the relationship of the variables, it can take any value from -1.00 to +1.00 , as long as the result obtained is close to these values it can be said that the correlation is perfect.

Statistically, there is a correlation of 0.982 , indicating that there is a strong positive correlation close to +1.00 . The $p$-value is $0.000<0.05$, therefore, $\mathrm{H}_{1}$ : Digital banking is significantly related to financial

Table 3. Normality Test.

\begin{tabular}{lcccccc}
\hline & \multicolumn{3}{c}{ Kolmogorov-Smirnova $^{\text {a }}$} & \multicolumn{3}{c}{ Shapiro-Wilk } \\
& Estadístico & gl & Sig. & Estadístico & gl & Sig. \\
\hline Penetración de la banca digital & 0.167 & 10 & $0.200^{*}$ & 0.931 & 10 & 0.455 \\
Inclusión financiera & 0.117 & 10 & $0.200^{*}$ & 0.961 & 10 & 0.795 \\
\hline
\end{tabular}

*. This is a lower bound of the true significance.

a. Lilliefors Significance Correction.

Source: Prepared by the author from SPSS-IBM. 
Table 4. Pearson's Correlation.

\begin{tabular}{llcc}
\hline & & Digital Banking Penetration & Financial Inclusion \\
\hline \multirow{3}{*}{ Digital Banking Penetration } & Pearson's Correlation & 1 & $0.982^{* *}$ \\
& Sig. (2-tailed) & 20 & 0.000 \\
& $\mathrm{~N}$ & $0.982^{* *}$ & 10 \\
\hline \multirow{3}{*}{ Financial Inclusion } & Pearson's Correlation & 0.000 & 1 \\
& Sig. & 10 & 10 \\
\hline
\end{tabular}

**. Correlation is significant at the 0.01 level (2-tailed).

Source: Prepared by the author from SPSS-IBM.

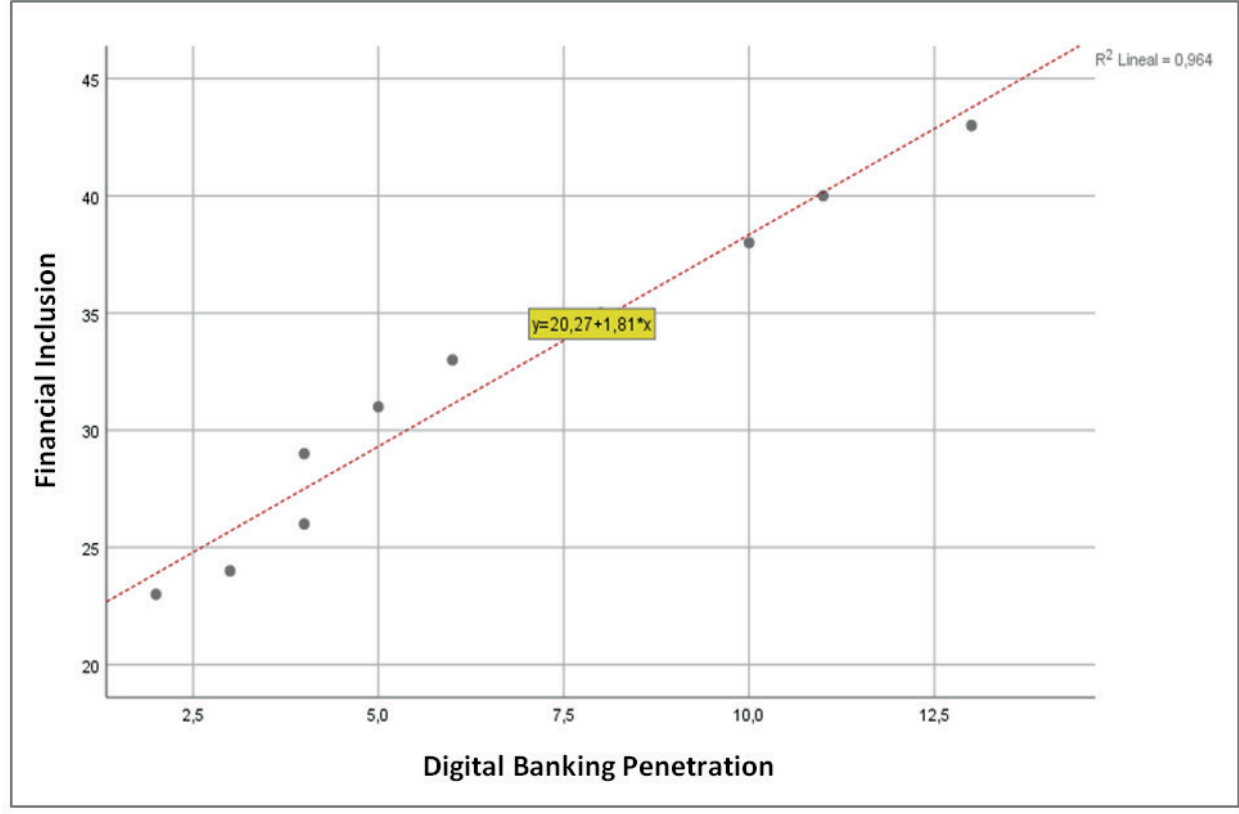

Figure 6. Diagrama de dispersión (correlación positiva). Fuente: Elaboración propia a partir de SPSS-IBM.

inclusion is accepted, because the variables are highly correlated. The statistical test was performed at $5 \%$ error or $95 \%$ confidence. The plot indicates that, for each percentage point increase in digital banking, financial inclusion increases by 1.81 percentage points.

The scatter plot indicates that, as digital banking transactions increase, financial inclusion also increases, a straight line with a positive slope.

\section{CONCLUSIONS}

As the study hypothesis has been proven, it can be stated that digital banking is significantly related to financial inclusion because both variables are highly correlated. Digital banking becomes a strategic alternative for financial inclusion in Peru, by using innovative technologies and software to optimize financial services and reach the most remote populations in the country in a cost-effective and inclusive manner.

Digital banking enables for the financial system to expand its services through internet platforms or cell phones, as it allows to open savings accounts, purchase insurance, apply for loans or credit cards, make investments in mutual funds, and conduct other transactions. However, as noted by the OAS (2018), the digital security incidents experienced by users of the banking system in Latin America are phishing by email (49.7\%), phishing by text message $(24.2 \%)$, phishing by phone call $(24.8 \%)$ and infection with malicious software or malware (35.67\%) 
In Peru, internet penetration was 53\% in 2018, 57\% in 2019 and $61 \%$ in 2020 , due to connectivity limitations. As a consequence, the handling of cash and its disadvantages such as: risk of theft, inappropriate data use, counterfeiting, fraud, money laundering, among others, are intensifying.

Traditional banking is facing great challenges such as digital transformation that requires investment in technology, change of culture, new business models, human resources, among others, in order to adapt to the current demands of the sector and market context.

\section{REFERENCES}

[1] Alonso, I., \& Carrio, A. (2019). La gestión de la información en banca: de las finanzas del comportamiento a la inteligencia artificial. Papeles de Economía Española (162), 148161. Retrieved from https://www.funcas.es/ revista/la-gestion-de-la-informacion-en-bancade-las-finanzas-del-comportamiento-a-lainteligencia-artificial-febrero-2020/

[2] Asobancaria (2017). La banca digital: estrategia clave para impulsar la inclusión financiera. Semana Económica. Retrieved from http://www. asobancaria.com/semanseconomicas/1074-a. pdf

[3] Azabache, C. (2018). La banca móvil como alternativa para la inclusión social en áreas rurales. Review of Global Management, 3(2), 67-81. Retrieved from https://dx.doi. org/10.19083/rgm.v3i2.779

[4] Banco Mundial. (2018). Inclusión financiera. Retrieved from https://www.bancomundial.org/ es/topic/financialinclusion/overview

[5] Bourgault, N., Egea, J., Guerrero, M., González, M., \& Mangin, J. (2013). Modelización de la confianza y seguridad en la adopción de la banca por internet. Revista Portuguesa de Marketing, 16(30), 35-54. Retrieved from https://search.proquest.com/scholarly-journals/ modelización-de-la-confianza-y-seguridad-en/ docview/1462485767/se-2?accountid=12268

[6] Bueno, E., Longo, M., \& Morcillo, P. (2017). La innovación del modelo de negocio bancario: El reto de la banca digital. Revista de la Asociación Española de Contabilidad y Administración de Empresas, 5-8. Retrieved from https://aeca.es/ wp-content/uploads/2019/10/120.pdf

[7] Comisión Económica para América Latina y el Caribe. (2020). Fortalecimiento de la inclusión y capacidades financieras en el ámbito rural. Retrieved from https://repositorio.cepal.org/ bitstream/handle/11362/45115/4/S2000323_ es.pdf

[8] Corporacion Andina de Fomento. (2020a). Las oportunidades de la digitalización en América Latina fente al Covid-19. Retrieved from https:// scioteca.caf.com/handle/123456789/1541

[9] Corporacion Andina de Fomento. (2020b). El estado de la digitalización de América Latina frente a la pandemia del COVID-19. Retrieved from https://scioteca.caf.com/ handle/123456789/1540

[10] Ditrendia. (2019). Mobile en España y en el Mundo 2019. Retrieved from https://ditrendia. es/informe-mobile-espana-mundo-2019/

[11] Federación Latinoamericana de Bancos. (2018). El blockchain y los smart contracts en la banca y el sector financiero. Retrieved from https://felaban.s3-us-west-2.amazonaws.com/ noticias/Noticia-2018-07-18.pdf

[12] Fondo Monetario Internacional. (2020). Financial Access Survey. Retrieved from https://data.imf.org/?sk=E5DCAB7E-A5CA4892-A6EA-598B5463A34C

[13] Global System for Mobile Communications. (2020). The mobile economy Latin America 2020. Retrieved from https://www.gsma.com/ mobileeconomy/wp-content/uploads/2020/12/ GSMA_MobileEconomy2020_LATAM_Eng.pdf

[14] Grupo Consultivo de Ayuda a los Pobres. (2015). Inclusión financiera digital: Implicancias para clientes, reguladores, supervisores $y$ organismos normativos. Retrieved from https:// www.cgap.org/sites/default/files/Brief-DigitalFinancial-Inclusion-Feb-2015-Spanish.pdf

[15] Ministerio de Transportes y Comunicaciones. (2021). MTC autoriza el despliegue de tecnología $5 G$ para servicios móviles. Retrieved from https://www.gob.pe/institucion/mtc/ noticias/482361-mtc-autoriza-el-desplieguede-tecnologia-5g-para-servicios-moviles

[16] Orazi, S., Martinez, L., \& Vigier, H. (2019). La inclusión financiera en América Latina y Europa. Ensayos de Economía, 29(55), 181204. Retrieved from https://doi.org/10.15446/ ede.v29n55.79425

[17] Organización de Estados Americanos. (2018). Estado de la Ciberseguridad en el Sector Bancario en América Latina y el Caribe. 
Retrieved from https://www.oas.org/es/sms/ cicte/sectorbancariospa.pdf

[18] Organización de las Naciones Unidas. (2020). Technology and Innovation Report. Retrieved from https://unctad.org/es/node/32191

[19] Revuelta, M. (2018). "Big Data": crisis y nuevos plantaeamientos en los flujos de comunicación de la cuarta revolución industrial. Área Abierta, 18(2), 309-324. Retrieved from: http://dx.doi. org/10.5209/ARAB.59521
[20] Serrano-Cobos, J. (2016). Tendencias tecnológicas en internet: Hacia un cambio de paradigma. El Profesional de la Información, 25(6), 843-850. Retrieved from http://dx.doi. org/10.3145/epi.2016.nov.01

[21] STATISTA. (2021). Digital Payments. Retrieved from https://www.statista.com/outlook/dmo/ fintech/digital-payments/south-america 